
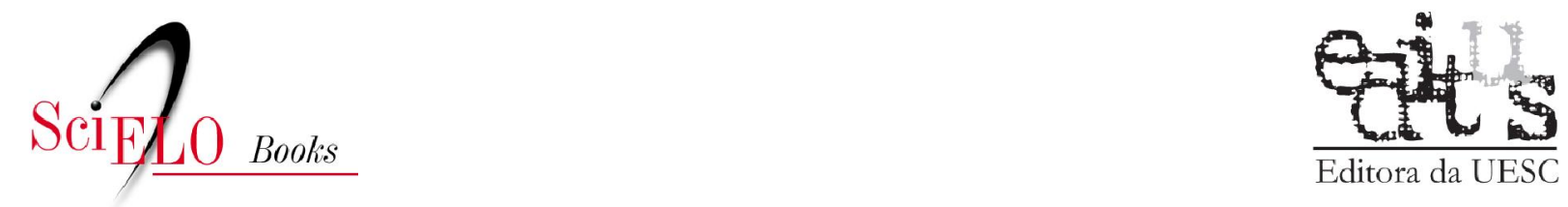

Editora da UESC

\title{
21 - Ecologia de poneromorfas em ambientes urbanos
}

\author{
Tercio da Silva Melo \\ Jacques H. C. Delabie
}

MELO, TS., and DELABIE, JH. Ecologia de poneromorfas em ambientes urbanos. In: DELABIE, JHC., et al., orgs. As formigas poneromorfas do Brasil [online]. Ilhéus, BA: Editus, 2015, pp. 313326. ISBN 978-85-7455-441-9. Available from SciELO Books $<$ http://books.scielo.org $>$.

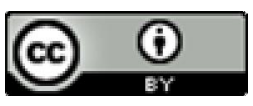

All the contents of this work, except where otherwise noted, is licensed under a Creative Commons Attribution 4.0 International license.

Todo o conteúdo deste trabalho, exceto quando houver ressalva, é publicado sob a licença Creative Commons Atribição 4.0.

Todo el contenido de esta obra, excepto donde se indique lo contrario, está bajo licencia de la licencia Creative Commons Reconocimento 4.0. 


\title{
21
}

\section{Ecologia de poneromorfas em ambientes urbanos}

\author{
Tercio da Silva Melo, Jacques H. C. Delabie
}

\section{Resumo}

Estudos avaliando os efeitos da urbanização sobre as comunidades de formigas são recentes, e ainda são poucos os trabalhos que utilizaram formigas como "ferramentas" de bioindicação para avaliar os efeitos da urbanização sobre a biodiversidade de uma forma geral. No Brasil, dentre os trabalhos realizados em ambientes urbanos, poucos são os que realmente avaliaram os efeitos da urbanização sobre as formigas, sendo majoritariamente levantamentos da mirmecofauna em diferentes tipos de habitats urbanos. Os estudos realizados em cidades brasileiras apresentaram formigas Poneromorfas, registrando mais de 70 espécies, sendo a Bahia (27 espécies) e a Mata Atlântica (48 espécies) respectivamente o estado e o bioma brasileiro que apresentaram maior riqueza específica. Entre as Poneromorfas, a família que apresenta maior riqueza é Ponerinae (47 espécies), seguido de Ectatomminae (13), Heteroponerinae (6), Amblyoponinae e Proceratiinae (com 1 espécie cada) e o gênero mais ricoé Hypoponera (15 espécies).No Brasil, as espécies Gnamptogenys striatula Mayr, 1884, Odontomachus chelifer (Latreille, 1802), Pachycondyla harpax (Fabricius, 1804), Pachycondyla striata (Smith, 1858) estão presentes em quase todas as cidades onde a fauna mirmecológica foi amostrada. Já as espécies
Ectatomma edentatum Roger, 1863, G. striatula, Hypoponera distinguenda (Emery, 1890), P. harpax e P. striata são as formigas encontradas em um mais amplo leque de habitats. Nas cidades brasileiras, dentre os diversos tipos de habitats urbanos já inventariados (casas, centros de reciclagem, colégios, fragmentos florestais, granja, hospitais, indústrias, jardins, parques, praças, quintais e terrenos baldios), casas foram os ambientes mais investigados, contudo estudos têm demonstrado que as áreas menos impactadas (como fragmentos florestais e parques) ainda conseguem manter um maior número de espécies nativas.

No que concerne a avaliação do efeito da urbanização brasileira sobre as Poneromorfas, características da atividade econômica (exemplo: cidades industriais e cidade cujo desenvolvimento econômico depende da agricultura) estão entre os primeiros fatores a influenciar a comunidade de formigas. Outros fatores que influenciam as comunidades de formigas são: (1) tamanho da cidade, (2) tempo de urbanização, (3) estrutura dos bairros, (4) presença de áreas de mata e (5) nível de conservação das residências, são aspectos ambientais determinantes da comunidade que ali vive. Ainda, moradias que apresentam jardins e quintais

MELO, Tercio da Silva; DELABIE, Jacques H. C. Ecologia de poneromorfas em ambientes urbanos.

In: DELABIE, Jacques H. C. et al. As formigas poneromorfas do Brasil. Ilhéus: Editus, 2015. p. 313-326. 
com presença de vegetação nativa possuem uma maior riqueza de espécies se comparadas a casas desprovidas desses ambientes. Já as áreas internas de casas apresentam maior abundância de formigas se comparado às áreas externas, apesar de serem espécies de formigas invasivas ou exóticas (de hábito generalista ou sinantrópico), as que dominam numericamente nas casas.

Espécies de formigas consideradas invasoras ou exóticas são dominantes nas cidades por explorarem melhor os recursos, estarem mais bem adaptadas às condições ambientais e por serem menos susceptíveis à predação. O principal impacto (negativo) destas formigas no ambiente urbano é a homogeneização biótica. Nas cidades brasileiras não há registro da ocorrência de espécies exóticas de formigas Poneromorfas (exemplo: Hypoponera punctatissima (Roger, 1859) e Hypoponera opacior Forel, 1893). Apesar de $H$. punctatissima e $H$. opacior, que foram introduzidas no Brasil, estarem presentes em ambientes antropizados, estas espécies não foram encontradas em ambiente urbano. Apesar de termos encontrado um grande número de espécies nativas de Poneromorfas listadas para cidades do Brasil, não encontramos referências que afirmam que alguma das formigas listadas nesse levantamento possua hábitos sinantrópicos. Contudo, propomos que as espécies E. edentatum, G. striatula, $H$. distinguenda, $P$. harpax e $P$. striata sejam consideradas sinantrópicas, devido à grande variedade de habitats urbanos que são capazes de ocupar.

\section{Abstract}

Ecology of poneromorph ants in urban environments - Studies evaluating the effects of urbanization on ant communities are a recent phenomenon, and there are few works using ants as bioindicator "tools" to assess the effects of urbanization on biodiversity in general. In Brazil, among those carried out in urban environments, few studies have specifically evaluated the effects of urbanization on ants, with most of them being ant fauna surveys in different types of urban habitats. Studies in Brazilian cities have revealed the occurrence of poneromorph ants, with more than 70 species being present, and with urban areas in Bahia (32 species) and in the Atlantic Rainforest zone (55 species) being respectively the state and biome that showed the higher species richness. Among the poneromorphs, the families that show the greatest richness are Ponerinae (47 species), followed by Ectatomminae (13), Heteroponerinae (6), Amblyoponinae and Proceratiinae (with 1 species each) and the richest genus is Pachycondyla (18 species). In Brazil, the ants Gnamptogenys striatula Mayr, 1884 Odontomachus chelifer (Latreille, 1802), Pachycondyla harpax (Fabricius, 1804), and Pachycondyla striata (Smith, 1858) are present in almost all cities where the ant fauna was sampled. However, Ectatomma edentatum Roger, 1863, G. striatula, Hypoponera distinguenda (Emery, 1890), P. harpax and P. striata are found in a wider range of habitats within the urban areas. In Brazilian towns, among the various types of urban habitats inventoried (houses, recycling centers, schools, forest fragments, farm, hospitals, industries, gardens, parks, squares, backyards and vacant lots), houses were the most investigated environments. However, studies have demonstrated that less impacted areas (such as parks and forest fragments) can still maintain a more native-like ant fauna.

Of the urbanization factors influencing Brazilian poneromorphs, economic activity of the city (e.g., industrial centers and towns whose economic development depends on agriculture) is one of the main factors influencing the ant community. Other factors influencing the ant communities are: (1) size of the city; (2) length of time since urbanization; (3) structure of neighborhoods; (4) presence of forest areas; and (5) level of preservation of the environment around the residences. Houses that have gardens and yards with native vegetation still present have a higher species richness compared to houses without these habitats. The internal areas of homes maintain a 
higher abundance of ants compared to external areas, although this is related to the presence of invasive or exotic species of ants (generalist or synanthropic species), which are numerically dominant in homes. These invasive native and exotic species are dominant in cities because they are better at exploiting the resources, are better adapted to urban environmental conditions and are less susceptible to predation. The main negative impact of these ants in the urban environment is biotic homogenization. In Brazilian cities, there is no record of alien species of poneromorphs ants (e.g., Hypoponera punctatissima (Roger, 1859) and
Hypoponera opacior Forel, 1893). Although these two species have been introduced into Brazil, and are present in anthropogenic environments, these ants were not found in urban environments. Although we found a large number of native poneromorph species listed for cities in Brazil, we did not find references establishing that the ants listed in the surveys have synanthropic habits. However, we propose that the species $E$. edentatum, G. striatula, $H$. distinguenda, $P$. harpax and $P$. striata can be considered as synanthropic due to the wide variety of urban habitats that they are able to occupy.

\section{Introdução}

As cidades agregam hoje em dia mais de 60\% da população humana mundial (ONU, 2011) e, especificamente no Brasil, mais de $80 \%$ da população vive em centros urbanos (IBGE, 2012). A formação e crescimento das cidades tornou-se um fenômeno global que tem se acentuado significativamente no último século (GRIMM et al., 2008; AGUILERA et al., 2011). Nesses centros urbanos, se concentram produção e consumo de bens (gerando descarte de resíduos), associados à gigantesca utilização de recursos naturais (PICKETT; CADENASSO, 2006). Tamanha interferência nas áreas nativas criadas pelas cidades leva a mudanças e problemas ambientais globais sérios (GRIMM et al., 2008), e o fenômeno urbano torna-se um campo de preocupação para a conservação ambiental (SHOCHAT et al., 2006). Dentre os problemas ambientais gerados pelas cidades, as principais alterações são causadas no uso/ cobertura do solo, nos ciclos bioquímicos, nas mudanças climáticas, nos sistemas hidrológicos e na manutenção da biodiversidade nativa frente às espécies introduzidas de outras regiões ou outros biomas (VITOUSEK et al., 1997; GRIMM et al., 2008).

Ambientes urbanos são facilmente caracterizáveis pela constante atividade humana, com altas densidades de população, adensamento de habitações, indústrias e comércios, escassez de áreas naturais, além de apresentar relativa impermeabilização dos solos, poluição, mudanças climáticas locais e uma elevada concentração de espécies exóticas (MCINTYRE et al., 2001). Ecologicamente, o processo de urbanização implica na conversão de áreas nativas em várias formas antropogênicas de uso da terra (PICKETT; CADENASSO 2006), forçando a fragmentação e isolamento de ambientes naturais, o aumento local na densidade do ser humano, levando à formação de "espaços urbanos" (MCINTYRE et al., 2000; MCINTYRE et al., 2001; PICKETT; CADENASSO, 2006).

No que concerne às mudanças na biodiversidade causadas pela urbanização, têm-se identificado alguns processos de alterações: através do aumento na produtividade primária, aumento na temperatura (SHOCHAT et al., 2006; KOWARIK, 2011), aumento na heterogeneidade/fragmentação e perturbação de habitat (KOWARIK, 2011), modificações na dinâmica trófica (SHOCHAT et al., 2006), degradação de áreas naturais e surgimento de novas áreas urbanizadas por justaposição às áreas já existentes (KOWARIK, 2011). De forma geral, a formação e expansão das cidades estão entre as principais causas de perda da biodiversidade nativa (MCKINNEY, 2002; MCKINNEY, 2006; FAETH et al., 2011) em escalas regionais e globais (GRIMM et al., 2008). Essa perda se dá devido as cidades criarem e/ou alterarem habitats, forçando adaptações morfológicas ou comportamentais em animais, nas dinâmicas populacionais, nas estruturas das comunidades de organismos (SHOCHAT et al., 2006; BYRNE, 
2007), além de beneficiarem poucas espécies melhor adaptadas à antropização do ambiente, promovendo a homogeneização biótica em diversas escalas espaciais (MCKINNEY, 2006).

Em contrapartida, estudos demonstram a importância das cidades na manutenção da biodiversidade (DEARBORN; KARK, 2010; KOWARIK, 2011), principalmente em habitats considerados como áreas verdes (quintais, jardins, praças, parques, fragmentos florestais e áreas de conservação ambiental). Cidades também podem ser ambientes climaticamente mais estáveis (quando localizados em regiões frias ou desérticas) e apresentar habitats antrópicos às vezes considerados análogos a habitats naturais (por exemplo, parques sombreados por árvores de grande porte), permitindo desta forma a permanência de espécies nativas ou mesmo consideradas raras e ameaçadas (LUNDHOLM; RICHARDSON, 2010; KOWARIK, 2011).

Dentre as vantagens de se conservar a biodiversidade nativa de ambientes urbanos, talvez, a manutenção dos serviços ecossistêmicos seja um dos fatores mais importantes para a garantia da qualidade de vida humana e ambiental (DEARBORN; KARK, 2010). Como os serviços ecossistêmicos são determinados pelas funções que as espécies exercem no meio ambiente, o número de espécies nativas pode ser considerado um indicativo confiável da manutenção destes serviços no ambiente urbano (SATTLER et al., 2010). Desta forma, alterações deletérias nas cidades podem significar perda de eficiência nos processos ecossistêmicos (ALBERTI, 2005). Por fim, esses padrões alterados de diversidade em áreas urbanas já são bem conhecidos em alguns grupos taxonômicos (ex.: plantas superiores, alguns grupos de artrópodes e aves), sobretudo nas regiões temperadas. No entanto, para uma maioria de grupos e regiões, há escassez de informações (FAETH et al., 2011).

\section{Poneromorfas urbanas}

Estudos avaliando os efeitos da urbanização sobre as comunidades de formigas são recentes, e ainda são poucos os trabalhos que utilizaram formigas como "ferramentas" de bioindicação para avaliar os efeitos da urbanização sobre a biodiversidade de uma forma geral (Tabela 21.I). Excluindo-se estudos realizados no Brasil, a maioria desses trabalhos foi realizada em regiões temperadas (principalmente nos Estados Unidos). Ainda, grande parte dos estudos tem comparado comunidades de formigas entre diferentes formas de habitats (tanto áreas naturais e não naturais) e alguns têm registrado espécies de formigas do grupo poneromorfas (Tabela 21.I).

No Brasil, dentre os trabalhos realizados em ambientes urbanos, poucos são os estudos

TABELA 21.I - Bibliografia temática sobre influência da urbanização sobre as assembleias de Formicidae em ambientes urbanos. Não foram incluídos estudos realizados no Brasil

\begin{tabular}{|c|c|c|c|}
\hline & TIPO DE ESTUDO & PAÍs & AUTOR \\
\hline \multirow{3}{*}{ 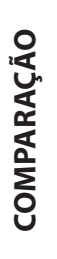 } & entre áreas naturais & Estados Unidos & Clarke et al, 2008; Ivanov; Keiper, 2010* \\
\hline & entre cidades & Rússia & Antonov, 2008 \\
\hline & entre formas de habitats & $\begin{array}{c}\text { Canadá, Estados Unidos, } \\
\text { Finlândia, Guiana Francesa, } \\
\text { Índia, Japão, México e } \\
\text { Trindade }\end{array}$ & $\begin{array}{l}\text { Yamaguchi, 2004*; Lessard; Buddle, 2005*; Kumar; } \\
\text { Mishra, 2008*; Vepsalainen et al., 2008; Delabie et } \\
\text { al., 2009*; Guzmán-Mendoza et al, 2010*; Uno et al., } \\
\text { 2010; Menke et al., 2011*; Wetterer; Dutra } 2011\end{array}$ \\
\hline \multirow{6}{*}{ 这 } & de espécies invasoras & Estados Unidos & $\begin{array}{l}\text { Holway; Suarez, 2006; Clarke et al, 2008; Menke et } \\
\text { al., 2010; Pecarevic et al., 2010*; Toennisson et al, } \\
\text { 2011*; Guérnard et al, 2014* }\end{array}$ \\
\hline & do efeito de borda & Canadá e Estados Unidos & Lessard; Buddle, 2005*; Ivanov; Keiper, 2010* \\
\hline & de gradiente de urbanização & Estados Unidos & $\begin{array}{l}\text { Sanford et al., 2009; Pecarevic et al., 2010*; Menke et } \\
\text { al., 2011*; Buczkowski; Richmond, } 2012\end{array}$ \\
\hline & de ocupação antrópica & Estados Unidos e Japão & Yamaguchi, 2004*; Buczkowski; Richmond, 2012 \\
\hline & da estrutura do habitat & Estados Unidos e Finlândia & $\begin{array}{l}\text { Clarke et al, 2008; Vepsalainen et al., 2008; Friedrich; } \\
\text { Philpott, 2009*; Toennisson et al, 2011* }\end{array}$ \\
\hline & de dinâmicas temporais & Estados Unidos e México & $\begin{array}{l}\text { Guzmán-Mendoza et al, 2010*; Buczkowski, 2011; } \\
\text { Buczkowski; Richmond, } 2012\end{array}$ \\
\hline
\end{tabular}

* Estudos onde ocorrem formigas poneromorfas. 
que realmente avaliaram os efeitos da urbanização sobre as formigas (MARQUES et al., 2002; SOARES et al., 2006; PACHECO; VASCONCELOS, 2007; MUNHAE et al., 2009; DÁTTILO et al., 2011; PIVA; CAMPOS, 2012; GUIMARÃES et al., 2013; CANTONE; CAMPOS, 2014), sendo grande parte dos estudos levantamentos da mirmecofauna em diferentes tipos de habitats urbanos (especialmente hospitais e domicílios). Pelo menos 20 estudos realizados em cidades brasileiras apresentaram formigas poneromorfas, registrando mais de 70 espécies (Tabela 21.II), sendo a Bahia (27 espécies) e a Mata Atlântica (48 espécies) respectivamente o estado e o bioma brasileiro que apresentaram maior riqueza específica. Entre as poneromorfas, a subfamília que apresenta maior riqueza é Ponerinae (47 espécies), seguida de Ectatomminae (13), Heteroponerinae (6), Amblyoponinae e Proceratiinae (com 1 espécie cada). Apesar do número expressivo de espécies registrado para regiões urbanas brasileiras e biomas, este ainda é um valor subestimado da riqueza, já que os estudos realizados se concentram em poucos estados brasileiros (apenas oito), e nos biomas da Amazônia, da Caatinga, do Cerrado e da Mata Atlântica. A maior parte dos estudos utilizou iscas para a atração de formigas (14 trabalhos), podendo representar um viés no registro das espécies de poneromorfas encontradas nas cidades.

No Brasil, as espécies Gnamptogenys striatula Mayr, 1884, Odontomachus chelifer (Latreille, 1802), Pachycondyla harpax (Fabricius, 1804), Pachycondyla striata (Smith, 1858) estão presentes em quase todas as cidades onde a fauna mirmecológica foi amostrada. Já as espécies Ectatomma edentatum Roger, 1863, G. striatula, Hypoponera distinguenda (Emery, 1890), P. harpax e P. striata são as formigas encontradas em um mais amplo leque de habitats. Nas cidades brasileiras, diversos tipos de habitats urbanos já foram inventariados: casas, centros de reciclagem, colégios, fragmentos florestais, granjas, hospitais, indústrias, jardins, parques, praças, quintais e terrenos baldios. Destes 13 tipos de habitats, as áreas domiciliares (casa, jardim e quintal) são os locais onde mais estudos foram realizados (10 artigos), mas fragmento florestal é o habitat que apresenta maior riqueza (46 espécies). Apesar de casas serem os ambientes mais investigados em cidades (exemplo: DELABIE et al., 1995; MARQUES et al., 2002; SOARES et al., 2006; FARNEDA et al., 2007; KAMURA et al., 2007; IOP et al., 2009; LOPES, 2009; PIVA;
CAMPOS, 2012), outros estudos têm demonstrado que áreas menos impactadas (como fragmentos florestais e parques) ainda conseguem manter um número consistente de espécies nativas (PACHECO; VASCONCELOS et al., 2007; GUIMARÃES et al., 2013; LUTINSKI et al., 2013; MELO et al., 2014). Em fragmentos florestais de São Paulo, as poneromorfas representaram uma grande porcentagem das formigas amostradas, demonstrando a importância de áreas naturais para a conservação da diversidade desse grupo (MORINI et al., 2007), e também estiveram entre os grupos com maior número de espécies das cidades do estado de Santa Catarina (LUTINSKI et al., 2013).

Ambientes naturais (como fragmentos florestais e parques) conseguem manter uma boa riqueza de poneromorfas porque apresentam diversas opções de locais para nidificação, que são importantes recursos para a conservação de formigas de forma geral (FRIEDRICH; PHILPOTT, 2009). Porém, o processo de urbanização em geral contribui a extirpar uma maioria desses sítios de estabelecimento de colônias, criando ao mesmo tempo locais de nidificação artificiais (exemplo: cavidades encontradas em construções) (FRIEDRICH; PHILPOTT, 2009). Talvez por esse motivo, conseguimos encontrar um número apreciável de espécies em habitats fortemente antropizados (por exemplo, foi encontrado um total de 38 espécies em casas, centros de reciclagem, colégios, granjas, hospitais, indústrias, jardins, praças, quintais e terrenos baldios), o que pode ser considerado próximo aos ambientes "mais" naturais encontrados na cidade (46 espécies) (Tabela 2). No mundo inteiro, os espaços verdes (terrenos abandonados, quintais, jardins e praças) são considerados importantes para a manutenção de uma fração considerável da mirmecofauna nativa (KAMURA et al., 2007; PACHECO; VASCONCELOS et al., 2007; MENKE et al., 2011; WENER, 2011; LUTINSKI et al., 2013; MELO et al., 2014), incluindo poneromorfas. Ainda, a importância desses espaços verdes vai além disso, já que podem servir também como corredores ecológicos para numerosos organismos, criando conectividade entre fragmentos florestais (MENKE et al., 2011).

No que concerne à avaliação do efeito da urbanização brasileira sobre as poneromorfas, características da atividade econômica da cidade (exemplo: cidades industriais e cidade cujo desenvolvimento econômico depende da agricultura) estão entre os primeiros fatores a influenciar a 
comunidade de formigas (MUNHAE et al., 2009). Provavelmente isso ocorra em função do fato deste tipo de economia gerar formas divergentes de exploração das áreas nativas e existir exigências legais distintas dos órgãos ambientais visando à conservação das áreas naturais. Outro fator que influencia as comunidades de formigas é o tamanho da cidade. Cidades consideradas grandes não apresentam variações temporais entre as poneromorfas, quando comparado a cidades pequenas (MUNHAE et al., 2009). Isto se deve às grandes cidades apresentarem menores variações climáticas (através da formação de ilhas de calor), não apresentando grandes variações de temperatura e umidade durante as estações do ano (MUNHAE et al., 2009). Isto torna as cidades grandes um ambiente mais estável (LUNDHOLM; RICHARDSON, 2010; KOWARIK, 2011) e favorável a uma determinada comunidade de formigas. Especificamente para poneromorfas, o período reprodutivo para o grupo em cidades se dá nos períodos mais quentes e chuvosos do ano (CANTONE; CAMPOS, 2014).

Dentro das cidades, a estrutura dos bairros e o nível de conservação das residências são os aspectos ambientais determinantes das espécies que podem ser encontradas (SOARES et al., 2006). Bairros recentemente urbanizados e que apresentam alto grau de perturbação antrópica favorecem o estabelecimento e permanência de espécies invasivas de formigas que passam a dominar o espaço (PIVA; CARVALHO CAMPOS, 2012). Em contrapartida, bairros localizados próximos a áreas de mata apresentam maior número de espécies nativas com uma menor abundância de indivíduos. Isso seja talvez umas das características mais importantes para a conservação de poneromorfas em ambientes urbanos (KAMURA et al., 2007; PACHECO; VASCONCELOS, 2007). Dentro de bairros, residências com jardins e quintais possuem uma maior riqueza de espécies se comparadas a casas desprovidas desses ambientes (PIVA; CARVALHO CAMPOS, 2012). Associado a isso, quando são mantidas plantas da vegetação nativa nesses jardins e quintais, estas geram impactos positivos na manutenção de poneromorfas (KAMURA et al., 2007). Já as áreas internas de casas apresentam maior abundância de formigas se comparado às áreas externas (KAMURA et al., 2007).

Dentro das casas, algumas poneromorfas (exemplo: Odontomachus e Gnamptogenys) são encontradas devido ao acesso permanente a alimentos, ausência de predadores e condições ambientais estáveis (MARQUES et al., 2002). Essas formigas são encontradas mais frequentemente nos interiores das casas durante a noite e em períodos chuvosos (MARQUES et al., 2002). A quantidade de espécies encontradas em residências está relacionada com o nível de conservação da casa. Residências bem conservadas apresentam menor número de espécies por oferecer menores oportunidades de nidificação às formigas (SOARES et al., 2006). Dentro das casas, a cozinha é onde podemos encontrar, em geral, a maior abundância e riqueza de formigas (MARQUES et al., 2002; KAMURA et al., 2007). Essa maior diversidade em cozinhas está relacionada com a maior disponibilidade de alimento, o que também favorece a manutenção de espécies invasoras (PIVA; CARVALHO CAMPOS, 2012). As formigas invasivas são espécies nativas de hábitos generalistas ou sinantrópicos, ou ainda espécies exóticas (DELABIE et al., 1995; MARQUES et al., 2002; SOARES et al., 2006; DÁTTILO et al., 2011), que predominam numericamente nas casas (DELABIE et al., 1995).

\section{Poneromorfas Sinantrópicas}

Dentre os trabalhos realizados acerca dos efeitos da urbanização sobre a comunidade de formigas, estudos avaliando os efeitos de espécies invasoras estão entre os mais frequentes (HOLWAY; SUAREZ, 2006; CLARKE et al, 2008; MENKE et al., 2010; PECAREVIC et al., 2010; TOENNISSON et al, 2011). Espécies de formigas consideradas invasoras urbanas podem ser nativas e exóticas de hábitos sinantrópicos, ou seja, espécies que são associadas a humanos e áreas urbanizadas (MCKINNEY, 2006). Ainda, essas formigas são dominantes nas cidades por explorarem melhor os recursos, estarem mais bem adaptadas às condições ambientais e por serem menos susceptíveis à predação (DELABIE et al., 1995; MARQUES et al., 2002; SHOCHAT et al., 2010; KOWARIK, 2011). O principal impacto (negativo) destas formigas no ambiente urbano é a homogeneização biótica, que ocorre quando espécies invasoras excluem competitivamente outras espécies nativas dos ambientes, levando outras espécies à extinção em diversas escalas espaciais (MCKINNEY, 2006). Muitas dessas espécies também trazem prejuízos à agricultura (DIEHL-FLEIG, 2006), além de serem vetores de patógenos aos humanos (ZARZUELA et al., 2002; PESQUERO et al., 2008; GARCIA et al., 2011; AQUINO et al., 2012; AQUINO et al., 2012). 
TABELA 21.II - Poneromorfas associadas à habitats urbanos no Brasil

\begin{tabular}{|c|c|c|c|c|c|}
\hline SUBFAMÍLIA & ESPÉCIE & ESTADO & BIOMA & HABITAT & AUTOR \\
\hline Amblyoponinae & Prionopelta sp. & Bahia (1 cidade) & Mata Atlântica & Jardim e quintal & Melo et al., 2014 \\
\hline \multirow[t]{22}{*}{ Ectatomminae } & $\begin{array}{c}\text { Ectatomma } \\
\text { edentatum Roger, } \\
1863\end{array}$ & $\begin{array}{l}\text { Minas Gerais e Santa } \\
\text { Catarina (1 cidade } \\
\text { cada), São Paulo (5 } \\
\text { cidades) }\end{array}$ & $\begin{array}{c}\text { Cerrado e Mata } \\
\text { Atlântica }\end{array}$ & $\begin{array}{c}\text { Centro de } \\
\text { reciclagem, colégio, } \\
\text { fragmento florestal, } \\
\text { hospital, parque e } \\
\text { praça }\end{array}$ & $\begin{array}{l}\text { Zarzuela et al., } \\
\text { 2002; Morini et } \\
\text { al., 2007; Pacheco; } \\
\text { Vasconcelos, 2007; } \\
\text { Munhae et al., 2009; } \\
\text { Souza et al., 2012; } \\
\text { Lutinski et al., 2013 }\end{array}$ \\
\hline & $\begin{array}{c}\text { Ectatomma muticum } \\
\text { Mayr, } 1870\end{array}$ & Bahia (4 cidades) & Mata Atlântica & $\begin{array}{c}\text { Fragmento florestal, } \\
\text { jardim, quintal e } \\
\text { terreno baldio }\end{array}$ & Melo et al., 2014 \\
\hline & $\begin{array}{c}\text { Ectatomma } \\
\text { opaciventris Roger, } \\
1861 \\
\end{array}$ & $\begin{array}{l}\text { Bahia e Minas Gerais } \\
\text { (1 cidade cada) }\end{array}$ & $\begin{array}{c}\text { Cerrado e Mata } \\
\text { Atlântica }\end{array}$ & $\begin{array}{l}\text { Fragmento florestal, } \\
\text { parque e praça }\end{array}$ & $\begin{array}{c}\text { Pacheco; } \\
\text { Vasconcelos, 2007; } \\
\text { Melo et al., } 2014\end{array}$ \\
\hline & $\begin{array}{c}\text { Ectatomma } \\
\text { permagnum Forel, } \\
1908 \\
\end{array}$ & $\begin{array}{c}\text { Minas Gerais ( } 1 \\
\text { cidade) }\end{array}$ & Cerrado & Parque & $\begin{array}{c}\text { Pacheco; } \\
\text { Vasconcelos, } 2007\end{array}$ \\
\hline & $\begin{array}{c}\text { Ectatomma } \\
\text { permagnum Forel, } \\
1908 \\
\end{array}$ & Paraná (1 cidade) & Mata Atlântica & Casa e indústria & $\begin{array}{l}\text { Oliveira; Campos- } \\
\text { Farinha, } 2005\end{array}$ \\
\hline & $\begin{array}{c}\text { Ectatomma sp. (1 } \\
\text { espécie) }\end{array}$ & Bahia (1 cidade) & Mata Atlântica & Fragmento florestal & $\begin{array}{l}\text { Guimarães et al., } \\
2013\end{array}$ \\
\hline & $\begin{array}{c}\text { Ectatomma sp. (1 } \\
\text { espécie) }\end{array}$ & São Paulo (1 cidade) & Mata Atlântica & Casa & Kamura et al., 2007 \\
\hline & $\begin{array}{c}\text { Ectatomma sp. (1 } \\
\text { espécie) }\end{array}$ & São Paulo (1 cidade) & Mata Atlântica & Casa & Piva; Campos, 2012 \\
\hline & $\begin{array}{c}\text { Ectatomma sp. (3 } \\
\text { espécies) }\end{array}$ & Bahia (1 cidade) & Mata Atlântica & $\begin{array}{l}\text { Ambiente urbano } \\
\text { não especificado }\end{array}$ & Delabie et al., 1999 \\
\hline & $\begin{array}{c}\text { Ectatomma sp. (3 } \\
\text { espécies) }\end{array}$ & São Paulo (1 cidade) & Mata Atlântica & Fragmento florestal & Dáttilo et al., 2011 \\
\hline & $\begin{array}{c}\text { Ectatomma } \\
\text { tuberculatum (Olivier, } \\
1791)\end{array}$ & Bahia (1 cidade) & Mata Atlântica & $\begin{array}{l}\text { Fragmento florestal e } \\
\text { terreno baldio }\end{array}$ & Melo et al., 2014 \\
\hline & $\begin{array}{c}\text { Gnamptogenys } \\
\text { menozzii (Borgmeier, } \\
1928) \\
\end{array}$ & Paraná (1 cidade) & Mata Atlântica & Casa e indústria & $\begin{array}{l}\text { Oliveira; Campos- } \\
\text { Farinha, } 2005\end{array}$ \\
\hline & $\begin{array}{c}\text { Gnamptogenys } \\
\text { regularis Mayr,1870 }\end{array}$ & $\begin{array}{c}\text { Minas Gerais (1 } \\
\text { cidade) }\end{array}$ & Cerrado & Parque & $\begin{array}{c}\text { Pacheco; } \\
\text { Vasconcelos, } 2007 \\
\end{array}$ \\
\hline & $\begin{array}{c}\text { Gnamptogenys sp. (1 } \\
\text { espécies) }\end{array}$ & Bahia (1 cidade) & Mata Atlântica & Casa & Delabie et al., 1995 \\
\hline & $\begin{array}{c}\text { Gnamptogenys sp. (1 } \\
\text { espécies) }\end{array}$ & São Paulo (1 cidade) & Mata Atlântica & Casa & Kamura et al., 2007 \\
\hline & $\begin{array}{c}\text { Gnamptogenys sp. (2 } \\
\text { espécies) }\end{array}$ & $\begin{array}{c}\text { Rio Grande do Sul (1 } \\
\text { cidade) }\end{array}$ & Pampa & Hospital & Garcia et al., 2011 \\
\hline & $\begin{array}{c}\text { Gnamptogenys sp. (3 } \\
\text { espécies) }\end{array}$ & Bahia (1 cidade) & Mata Atlântica & $\begin{array}{l}\text { Ambiente urbano } \\
\text { não especificado }\end{array}$ & Delabie et al., 1999 \\
\hline & $\begin{array}{c}\text { Gnamptogenys sp. (5 } \\
\text { espécies) }\end{array}$ & São Paulo (1 cidade) & Mata Atlântica & Casa & Souza et al., 2012 \\
\hline & $\begin{array}{c}\text { Gnamptogenys } \\
\text { striatula Mayr, } 1884\end{array}$ & $\begin{array}{l}\text { Amazonas e Minas } \\
\text { Gerais ( } 1 \text { cidade } \\
\text { cada), Bahia ( } 2 \\
\text { cidades), São Paulo } \\
\text { (4 cidades), Santa } \\
\text { Catarina (10 cidades) }\end{array}$ & $\begin{array}{c}\text { Cerrado, Floresta } \\
\text { Amazônica e Mata } \\
\text { Atlântica }\end{array}$ & $\begin{array}{l}\text { Casa, centro de } \\
\text { reciclagem, colégio, } \\
\text { fragmento florestal, } \\
\text { parque e praça }\end{array}$ & $\begin{array}{l}\text { Marques et al., } \\
\text { 2002; Morini et } \\
\text { al., 2007; Pacheco; } \\
\text { Vasconcelos, 2007; } \\
\text { Iop et al., 2009; } \\
\text { Munhae et al., 2009; } \\
\text { Souza et al., 2012; } \\
\text { Lutinski et al., 2013; } \\
\text { Melo et al., 2014 }\end{array}$ \\
\hline & $\begin{array}{c}\text { Gnamptogenys } \\
\text { sulcata (Smith, 1858) }\end{array}$ & $\begin{array}{c}\text { Bahia e Santa } \\
\text { Catarina ( } 1 \text { cidade } \\
\text { cada) }\end{array}$ & Mata Atlântica & $\begin{array}{c}\text { Centro de } \\
\text { reciclagem, colégio, } \\
\text { fragmento florestal e } \\
\text { parque }\end{array}$ & $\begin{array}{l}\text { Lutinski et al., 2013; } \\
\text { Melo et al., } 2014\end{array}$ \\
\hline & Typhlomyrmex sp. & Paraná (1 cidade) & Mata Atlântica & Casa e indústria & $\begin{array}{l}\text { Oliveira; Campos- } \\
\text { Farinha, } 2005 \\
\end{array}$ \\
\hline & $\begin{array}{c}\text { Typhlomyrmex } \\
\text { rogenhoferi Mayr, } \\
1862 \\
\end{array}$ & São Paulo (1 cidade) & Mata Atlântica & Fragmento florestal & Souza et al., 2012 \\
\hline
\end{tabular}




\begin{tabular}{|c|c|c|c|c|c|}
\hline SUBFAMÍLIA & ESPÉCIE & ESTADO & BIOMA & HABITAT & AUTOR \\
\hline \multirow[t]{6}{*}{ Heteroponerinae } & $\begin{array}{c}\text { Heteroponera } \\
\text { dentinodis (Mayr, } \\
1887 \text { ) } \\
\end{array}$ & São Paulo (1 cidade) & Mata Atlântica & Fragmento florestal & Morini et al., 2007 \\
\hline & $\begin{array}{c}\text { Heteroponera dolo } \\
\text { (Roger, 1860) }\end{array}$ & São Paulo (1 cidade) & Mata Atlântica & Fragmento florestal & Morini et al., 2007 \\
\hline & $\begin{array}{c}\text { Heteroponera flava } \\
\text { Kempf, } 1962\end{array}$ & $\begin{array}{c}\text { Santa Catarina (4 } \\
\text { cidades) }\end{array}$ & Mata Atlântica & $\begin{array}{c}\text { Centro de } \\
\text { reciclagem, colégio, } \\
\text { fragmento florestal e } \\
\text { parque }\end{array}$ & Lutinski et al., 2013 \\
\hline & $\begin{array}{l}\text { Heteroponera inermis } \\
\quad(\text { Emery, 1894) }\end{array}$ & $\begin{array}{c}\text { Santa Catarina ( } 3 \\
\text { cidades) }\end{array}$ & Mata Atlântica & $\begin{array}{c}\text { Centro de } \\
\text { reciclagem, colégio, } \\
\text { fragmento florestal e } \\
\text { parque }\end{array}$ & Lutinski et al., 2013 \\
\hline & $\begin{array}{c}\text { Heteroponera mayri } \\
\text { (Kempf, 1962) }\end{array}$ & São Paulo (1 cidade) & Mata Atlântica & Fragmento florestal & Morini et al., 2007 \\
\hline & Heteroponera sp. & São Paulo (1 cidade) & Mata Atlântica & Casa & Kamura et al., 2007 \\
\hline \multirow[t]{20}{*}{ Ponerinae } & $\begin{array}{c}\text { Anochetus } \\
\text { altisquamis Mayr, } \\
1887 \\
\end{array}$ & São Paulo (1 cidade) & Mata Atlântica & $\begin{array}{c}\text { Casa e fragmento } \\
\text { florestal }\end{array}$ & Souza et al., 2012 \\
\hline & $\begin{array}{c}\text { Anochetus neglectus } \\
\text { Emery, } 1894\end{array}$ & $\begin{array}{c}\text { Minas Gerais ( } 1 \\
\text { cidade) e São Paulo } \\
\text { ( } 2 \text { cidades })\end{array}$ & $\begin{array}{c}\text { Cerrado e Mata } \\
\text { Atlântica }\end{array}$ & Parque e praça & $\begin{array}{c}\text { Pacheco; } \\
\text { Vasconcelos, 2007; } \\
\text { Munhae et al., } 2009\end{array}$ \\
\hline & $\begin{array}{c}\text { Anochetus simoni } \\
\text { Emery, } 1890\end{array}$ & Bahia (1 cidade) & Mata Atlântica & Fragmento florestal & Melo et al., 2014 \\
\hline & $\begin{array}{c}\text { Anochetus sp. (1 } \\
\text { espécie) }\end{array}$ & Bahia (1 cidade) & Mata Atlântica & Fragmento florestal & $\begin{array}{c}\text { Guimarães et al., } \\
2013 \\
\end{array}$ \\
\hline & $\begin{array}{c}\text { Anochetus sp. (1 } \\
\text { espécie) }\end{array}$ & São Paulo (1 cidade) & Mata Atlântica & Fragmento florestal & $\begin{array}{c}\text { Cantone; Campos, } \\
2014 \\
\end{array}$ \\
\hline & $\begin{array}{c}\text { Anochetus targionii } \\
\text { Emery, } 1894\end{array}$ & Bahia (1 cidade) & Mata Atlântica & $\begin{array}{c}\text { Fragmento florestal, } \\
\text { jardim, quintal e } \\
\text { terreno baldio }\end{array}$ & Melo et al., 2014 \\
\hline & $\begin{array}{c}\text { Dinoponera australis } \\
\text { Emery, } 1901\end{array}$ & $\begin{array}{c}\text { Santa Catarina ( } 2 \\
\text { cidades) }\end{array}$ & Mata Atlântica & $\begin{array}{c}\text { Colégio, parque, } \\
\text { centro de reciclagem } \\
\text { e fragmento florestal }\end{array}$ & Lutinski et al., 2013 \\
\hline & $\begin{array}{c}\text { Dinoponera } \\
\text { quadriceps Santschi, } \\
1921\end{array}$ & Bahia & Caatinga & Universidade & Delabie, obs. pess. \\
\hline & $\begin{array}{c}\text { Hypoponera } \\
\text { distinguenda (Emery, } \\
1890)\end{array}$ & $\begin{array}{c}\text { Santa Catarina ( } 2 \\
\text { cidades) }\end{array}$ & Mata Atlântica & $\begin{array}{l}\text { Casa, centro de } \\
\text { reciclagem, colégio, } \\
\text { comércio, fragmento } \\
\text { florestal e parque }\end{array}$ & $\begin{array}{l}\text { Farneda et al., 2007; } \\
\text { Lutinski et al., } 2013\end{array}$ \\
\hline & $\begin{array}{c}\text { Hypoponera foreli } \\
\text { Mayr, } 1887\end{array}$ & Bahia (2 cidades) & Mata Atlântica & Fragmento florestal & Melo et al., 2014 \\
\hline & $\begin{array}{c}\text { Hypoponera } \\
\text { opaciceps (Mayr, } \\
1887)\end{array}$ & $\begin{array}{c}\text { Santa Catarina (1 } \\
\text { cidade) }\end{array}$ & Mata Atlântica & $\begin{array}{c}\text { Centro de } \\
\text { reciclagem, colégio, } \\
\text { fragmento florestal e } \\
\text { parque }\end{array}$ & Lutinski et al., 2013 \\
\hline & $\begin{array}{c}\text { Hypoponera } \\
\text { punctatissima (Roger, } \\
1859 \text { ) }\end{array}$ & São Paulo (1 cidade) & Cerrado & Granja & Delabie; Blard, 2002 \\
\hline & $\begin{array}{c}\text { Hypoponera sp. (1 } \\
\text { espécie) }\end{array}$ & Bahia (1 cidade) & Mata Atlântica & Fragmento florestal & $\begin{array}{l}\text { Guimarães et al., } \\
2013\end{array}$ \\
\hline & $\begin{array}{c}\text { Hypoponera sp. (1 } \\
\text { espécie) }\end{array}$ & Bahia (1 cidade) & Mata Atlântica & $\begin{array}{l}\text { Ambiente urbano } \\
\text { não especificado }\end{array}$ & Delabie et al., 1999 \\
\hline & $\begin{array}{c}\text { Hypoponera sp. (1 } \\
\text { espécie) }\end{array}$ & Goiás (1 cidade) & Cerrado & Hospital & Pasqueiro et al., 2008; \\
\hline & $\begin{array}{c}\text { Hypoponera sp. (1 } \\
\text { espécie) }\end{array}$ & $\begin{array}{c}\text { Rio Grande do Sul (1 } \\
\text { cidade) }\end{array}$ & Mata Atlântica & Hospital & Garcia et al., 2011 \\
\hline & $\begin{array}{l}\text { Hypoponera sp. (1 } \\
\text { espécie) }\end{array}$ & $\begin{array}{c}\text { Santa Catarina (1 } \\
\text { cidade) }\end{array}$ & Mata Atlântica & $\begin{array}{c}\text { Centro de } \\
\text { reciclagem, colégio, } \\
\text { fragmento florestal e } \\
\text { parque }\end{array}$ & Lutinski et al., 2013 \\
\hline & $\begin{array}{c}\text { Hypoponera sp. (1 } \\
\text { espécie) }\end{array}$ & São Paulo (1 cidade) & Mata Atlântica & Casa & Piva; Campos, 2012 \\
\hline & $\begin{array}{l}\text { Hypoponera sp. (1 } \\
\text { espécie) }\end{array}$ & São Paulo (1 cidade) & Mata Atlântica & Casa & Lopes, 2009 \\
\hline & $\begin{array}{c}\text { Hypoponera sp. (1 } \\
\text { espécie) }\end{array}$ & São Paulo (3 cidades) & Mata Atlântica & Parque e praça & Munhae et al., 2009 \\
\hline
\end{tabular}




\begin{tabular}{|c|c|c|c|c|c|}
\hline SUBFAMÍLIA & ESPÉCIE & ESTADO & BIOMA & HABITAT & AUTOR \\
\hline & $\begin{array}{c}\text { Hypoponera spp. (2 } \\
\text { espécies) }\end{array}$ & São Paulo (1 cidade) & Mata Atlântica & Casa & Kamura et al., 2007 \\
\hline & $\begin{array}{c}\text { Hypoponera spp. (2 } \\
\text { espécies) }\end{array}$ & $\begin{array}{c}\text { Santa Catarina (1 } \\
\text { cidade) }\end{array}$ & Mata Atlântica & Casa e comércio & lop et al., 2009 \\
\hline & $\begin{array}{c}\text { Hypoponera spp. (3 } \\
\text { espécies) }\end{array}$ & São Paulo (1 cidade) & Mata Atlântica & $\begin{array}{c}\text { Casa e fragmento } \\
\text { florestal }\end{array}$ & Souza et al., 2012 \\
\hline & $\begin{array}{c}\text { Hypoponera spp. (6 } \\
\text { espécies) }\end{array}$ & Bahia (5 cidades) & Mata Atlântica & $\begin{array}{l}\text { Casa, fragmento } \\
\text { florestal, jardim, } \\
\text { quintal e terreno } \\
\text { baldio }\end{array}$ & Melo et al., 2014 \\
\hline & $\begin{array}{c}\text { Hypoponera spp. (9 } \\
\text { espécies) }\end{array}$ & São Paulo (1 cidade) & Mata Atlântica & Fragmento florestal & Morini et al., 2007 \\
\hline & $\begin{array}{c}\text { Hypoponera trigona } \\
\text { (Mayr, 1887) }\end{array}$ & $\begin{array}{c}\text { Santa Catarina ( } 3 \\
\text { cidades) }\end{array}$ & Mata Atlântica & $\begin{array}{c}\text { Centro de } \\
\text { reciclagem, colégio, } \\
\text { fragmento florestal e } \\
\text { parque }\end{array}$ & Lutinski et al., 2013 \\
\hline & $\begin{array}{c}\text { Leptogenys arcuata } \\
\text { Roger, } 1861\end{array}$ & Bahia (1 cidade) & Mata Atlântica & $\begin{array}{c}\text { Jardim, quintal e } \\
\text { terreno baldio }\end{array}$ & Melo et al., 2014 \\
\hline & $\begin{array}{c}\text { Leptogenys boh/si } \\
\text { Emery, } 1896\end{array}$ & Bahia (1 cidade) & Mata Atlântica & Fragmento florestal & Melo et al., 2014 \\
\hline & $\begin{array}{c}\text { Leptogenys sp. (1 } \\
\text { espécie) }\end{array}$ & São Paulo (1 cidade) & Mata Atlântica & Fragmento florestal & Morini et al., 2007 \\
\hline & $\begin{array}{l}\text { Leptogenys sp. (1 } \\
\text { espécie) }\end{array}$ & Bahia (1 cidade) & Mata Atlântica & $\begin{array}{l}\text { Ambiente urbano } \\
\text { não especificado }\end{array}$ & Delabie et al., 1999 \\
\hline & $\begin{array}{c}\text { Mayaponera } \\
\text { constricta (Mayr, } \\
1884 \text { ) }\end{array}$ & $\begin{array}{l}\text { Bahia ( } 3 \text { cidades) e } \\
\text { São Paulo ( } 1 \text { cidade) }\end{array}$ & Mata Atlântica & Fragmento florestal & $\begin{array}{l}\text { Morini et al, 2007; } \\
\text { Melo et al., } 2014\end{array}$ \\
\hline & $\begin{array}{c}\text { Neoponera apicalis } \\
\text { (Latreille, 1802) }\end{array}$ & Bahia (3 cidades) & Mata Atlântica & Fragmento florestal & Melo et al., 2014 \\
\hline & $\begin{array}{c}\text { Neoponera concava } \\
\text { (MacKay; MacKay, } \\
\text { 2010) }\end{array}$ & Bahia (2 cidades) & Mata Atlântica & Fragmento florestal & Melo et al., 2014 \\
\hline & $\begin{array}{c}\text { Neoponera crenata } \\
\text { (Roger, 1861) }\end{array}$ & $\begin{array}{c}\text { Santa Catarina (6 } \\
\text { cidades) e São Paulo } \\
\text { (1 cidade) }\end{array}$ & Mata Atlântica & $\begin{array}{c}\text { Centro de } \\
\text { reciclagem, colégio, } \\
\text { fragmento florestal, } \\
\text { parque e praça }\end{array}$ & $\begin{array}{l}\text { Munhae et al., 2009; } \\
\text { Lutinski et al., } 2013\end{array}$ \\
\hline & $\begin{array}{c}\text { Neoponera laevigata } \\
\text { Smith, } 1858\end{array}$ & $\begin{array}{c}\text { Minas Gerais (1 } \\
\text { cidade) }\end{array}$ & Cerrado & Parque & $\begin{array}{c}\text { Pacheco; } \\
\text { Vasconcelos, } 2007\end{array}$ \\
\hline & $\begin{array}{l}\text { Neoponera marginata } \\
\text { (Roger, 1861) }\end{array}$ & $\begin{array}{c}\text { Santa Catarina (1 } \\
\text { cidade) }\end{array}$ & Mata Atlântica & $\begin{array}{c}\text { Centro de } \\
\text { reciclagem, colégio, } \\
\text { fragmento florestal e } \\
\text { parque }\end{array}$ & Lutinski et al., 2013 \\
\hline & $\begin{array}{l}\text { Neoponera verenae } \\
\text { (Forel, 1922) }\end{array}$ & $\begin{array}{c}\text { Bahia ( } 2 \text { cidades) } \\
\text { e Minas Gerais ( } 1 \\
\text { cidade) }\end{array}$ & $\begin{array}{l}\text { Cerrado e Mata } \\
\text { Atlântica }\end{array}$ & $\begin{array}{c}\text { Fragmento florestal e } \\
\text { parque }\end{array}$ & $\begin{array}{c}\text { Pacheco; } \\
\text { Vasconcelos, 2007; } \\
\text { Melo et al., } 2014 \\
\end{array}$ \\
\hline & $\begin{array}{c}\text { Neoponera villosa } \\
\text { (Fabricius, 1804) }\end{array}$ & $\begin{array}{c}\text { Minas Gerais ( } 1 \\
\text { cidade) e Santa } \\
\text { Catarina (4 cidades) }\end{array}$ & $\begin{array}{l}\text { Cerrado e Mata } \\
\text { Atlântica }\end{array}$ & $\begin{array}{c}\text { Centro de } \\
\text { reciclagem, colégio, } \\
\text { fragmento florestal, } \\
\text { parque e praça }\end{array}$ & $\begin{array}{c}\text { Pacheco; } \\
\text { Vasconcelos, 2007; } \\
\text { Lutinski et al., } 2013\end{array}$ \\
\hline & $\begin{array}{l}\text { Odontomachus affinis } \\
\text { (Guérin-ménenville, } \\
1844)\end{array}$ & São Paulo (2 cidades) & Mata Atlântica & $\begin{array}{l}\text { Casa, fragmento } \\
\text { florestal e praça }\end{array}$ & $\begin{array}{c}\text { Morini et al., 2007; } \\
\text { Munhae et al., 2009; } \\
\text { Souza et al., } 2012\end{array}$ \\
\hline & $\begin{array}{c}\text { Odontomachus bauri } \\
\text { Emery, } 1892\end{array}$ & $\begin{array}{c}\text { Bahia (2 cidades), } \\
\text { Amazonas, Minas } \\
\text { Gerais e São Paulo (1 } \\
\text { cidade cada) }\end{array}$ & $\begin{array}{c}\text { Cerrado, Floresta } \\
\text { Amazônica e Mata } \\
\text { Atlântica }\end{array}$ & $\begin{array}{c}\text { Casa, fragmento } \\
\text { florestal, jardim, } \\
\text { quintal e terreno } \\
\text { baldio } \\
\end{array}$ & $\begin{array}{c}\text { Marques et al., 2002; } \\
\text { Soares et al., 2006; } \\
\text { Melo et al., } 2014\end{array}$ \\
\hline & $\begin{array}{c}\text { Odontomachus } \\
\text { brunneus (Patton, } \\
1894)\end{array}$ & Bahia (2 cidades) & Mata Atlântica & $\begin{array}{l}\text { Fragmento florestal, } \\
\text { jardim e quintal }\end{array}$ & Melo et al., 2014 \\
\hline & $\begin{array}{c}\text { Odontomachus } \\
\text { chelifer (Latreille, } \\
\text { 1802) }\end{array}$ & $\begin{array}{c}\text { Minas Gerais (1 } \\
\text { cidade), São Paulo } \\
\text { (2 cidades) e Santa } \\
\text { Catarina ( } 8 \text { cidades) }\end{array}$ & $\begin{array}{l}\text { Cerrado e Mata } \\
\text { Atlântica }\end{array}$ & $\begin{array}{l}\text { Casa, centro de } \\
\text { reciclagem, colégio, } \\
\text { fragmento florestal, } \\
\text { parque e praça }\end{array}$ & $\begin{array}{c}\text { Morini et al., } \\
\text { 2007; Pacheco; } \\
\text { Vasconcelos, 2007; } \\
\text { Munhae et al., 2009; } \\
\text { Souza et al., 2012; } \\
\text { Lutinski et al., 2013 }\end{array}$ \\
\hline & $\begin{array}{c}\text { Odontomachus } \\
\text { haematodus } \\
\text { (Linnaeus, 1758) }\end{array}$ & Bahia (3 cidades) & Mata Atlântica & $\begin{array}{c}\text { Fragmento florestal, } \\
\text { jardim, quintal e } \\
\text { terreno baldio }\end{array}$ & Melo et al., 2014 \\
\hline
\end{tabular}




\begin{tabular}{|c|c|c|c|c|c|}
\hline SUBFAMÍLIA & ESPÉCIE & ESTADO & BIOMA & HABITAT & AUTOR \\
\hline & $\begin{array}{c}\text { Odontomachus } \\
\text { meinerti Forel, } 1905\end{array}$ & $\begin{array}{l}\text { Bahia (5 cidades), } \\
\text { São Paulo ( } 2 \text { cidades) } \\
\text { e Minas Gerais ( } 1 \\
\text { cidade) }\end{array}$ & $\begin{array}{c}\text { Cerrado e Mata } \\
\text { Atlântica }\end{array}$ & $\begin{array}{c}\text { Casa, fragmento } \\
\text { florestal, parque e } \\
\text { praça }\end{array}$ & $\begin{array}{c}\text { Morini et al., } \\
\text { 2007; Pacheco; } \\
\text { Vasconcelos, 2007; } \\
\text { Souza et al., 2012; } \\
\text { Melo et al., } 2014\end{array}$ \\
\hline & $\begin{array}{c}\text { Odontomachus sp. (1 } \\
\text { espécie) }\end{array}$ & Bahia (1 cidade) & Mata Atlântica & Casa & Delabie et al, 1995 \\
\hline & $\begin{array}{c}\text { Odontomachus sp. (1 } \\
\text { espécie) }\end{array}$ & Bahia (1 cidade) & Mata Atlântica & Fragmento florestal & $\begin{array}{l}\text { Guimarães et al., } \\
2013\end{array}$ \\
\hline & $\begin{array}{c}\text { Odontomachus sp. (1 } \\
\text { espécie) }\end{array}$ & Bahia (1 cidade) & Mata Atlântica & $\begin{array}{l}\text { Ambiente urbano } \\
\text { não especificado }\end{array}$ & Delabie et al., 1999 \\
\hline & $\begin{array}{c}\text { Odontomachus sp. (1 } \\
\text { espécie) }\end{array}$ & $\begin{array}{c}\text { Minas Gerais (1 } \\
\text { cidade), }\end{array}$ & Cerrado & $\begin{array}{c}\text { Fragmento florestal, } \\
\text { parque e praça }\end{array}$ & $\begin{array}{c}\text { Pacheco; } \\
\text { Vasconcelos, } 2007\end{array}$ \\
\hline & $\begin{array}{c}\text { Odontomachus sp. (1 } \\
\text { espécie) }\end{array}$ & Paraná (1 cidade) & Mata Atlântica & Casa e indústria & $\begin{array}{l}\text { Oliveira; Campos- } \\
\text { Farinha, } 2005\end{array}$ \\
\hline & $\begin{array}{c}\text { Odontomachus sp. (1 } \\
\text { espécie) }\end{array}$ & São Paulo (1 cidade) & Mata Atlântica & Fragmento florestal & Dáttilo et al., 2011 \\
\hline & $\begin{array}{c}\text { Odontomachus sp. (1 } \\
\text { espécie) }\end{array}$ & São Paulo (1 cidade) & Mata Atlântica & Fragmento florestal & Kamura et al., 2007 \\
\hline & $\begin{array}{c}\text { Pachycondyla } \\
\text { crassinoda (Latreille, } \\
1802 \text { ) }\end{array}$ & Bahia (4 cidades) & Mata Atlântica & Fragmento florestal & Melo et al., 2014 \\
\hline & $\begin{array}{l}\text { Pachycondyla harpax } \\
\text { (Fabricius, 1804) }\end{array}$ & $\begin{array}{c}\text { Bahia (2 cidades), } \\
\text { Minas Gerais ( } 1 \\
\text { cidade) e Santa } \\
\text { Catarina ( } 9 \text { cidades) }\end{array}$ & $\begin{array}{c}\text { Cerrado e Mata } \\
\text { Atlântica }\end{array}$ & $\begin{array}{l}\text { Casa, centro de } \\
\text { reciclagem, colégio, } \\
\text { fragmentos florestais, } \\
\text { jardim, parques, } \\
\text { praça, quintal, e } \\
\text { terreno baldio }\end{array}$ & $\begin{array}{c}\text { Pacheco; } \\
\text { Vasconcelos, 2007; } \\
\text { lop et al., 2009; } \\
\text { Lutinski et al., 2013; } \\
\text { Melo et al., } 2014\end{array}$ \\
\hline & $\begin{array}{c}\text { Pachycondyla } \\
\text { impressa Roger, } 1861\end{array}$ & Bahia (1 cidade) & Mata Atlântica & $\begin{array}{c}\text { Fragmento florestal e } \\
\text { terreno baldio }\end{array}$ & Melo et al., 2014 \\
\hline & $\begin{array}{l}\text { Pachycondyla striata } \\
\text { (Smith, 1858) }\end{array}$ & $\begin{array}{c}\text { Minas Gerais ( } 1 \\
\text { cidade), São Paulo } \\
\text { (4 cidades), Santa } \\
\text { Catarina (10 cidades) }\end{array}$ & $\begin{array}{c}\text { Cerrado e Mata } \\
\text { Atlântica }\end{array}$ & $\begin{array}{l}\text { Casa, centro de } \\
\text { reciclagem, colégio, } \\
\text { comércio, fragmento } \\
\text { florestal, parque e } \\
\text { praça }\end{array}$ & $\begin{array}{c}\text { Farneda et al., } \\
\text { 2007; Morini et } \\
\text { al., 2007; Pacheco; } \\
\text { Vasconcelos, 2007; } \\
\text { lop et al., 2009; } \\
\text { Munhae et al., 2009; } \\
\text { Souza et al., 2012; } \\
\text { Lutinski et al., 2013 }\end{array}$ \\
\hline & $\begin{array}{c}\text { Ponerinae } \\
\text { ('Pachycondyla'sp.) (1 } \\
\text { espécie) }\end{array}$ & Bahia (1 cidade) & Mata Atlântica & Fragmento florestal & Melo et al., 2014 \\
\hline & $\begin{array}{c}\text { Ponerinae } \\
\text { ('Pachycondyla'sp.) (1 } \\
\text { espécie) }\end{array}$ & $\begin{array}{l}\text { Rio Grande do Sul (1 } \\
\text { cidade) }\end{array}$ & Mata Atlântica & Hospital & Garcia et al., 2011 \\
\hline & $\begin{array}{c}\text { Ponerinae } \\
\text { ('Pachycondyla'sp.) (1 } \\
\text { espécie) }\end{array}$ & $\begin{array}{c}\text { Santa Catarina ( } 1 \\
\text { cidade) }\end{array}$ & Mata Atlântica & Casa e comércio & Farneda et al., 2007 \\
\hline & $\begin{array}{c}\text { Ponerinae } \\
\text { ('Pachycondyla'sp.) (1 } \\
\text { espécie) }\end{array}$ & $\begin{array}{c}\text { Santa Catarina ( } 4 \\
\text { cidades) }\end{array}$ & Mata Atlântica & $\begin{array}{c}\text { Centro de } \\
\text { reciclagem, colégio, } \\
\text { fragmento florestal e } \\
\text { parque }\end{array}$ & Lutinski et al., 2013 \\
\hline & $\begin{array}{c}\text { Ponerinae } \\
\text { ('Pachycondyla'sp.) (1 } \\
\text { espécie) }\end{array}$ & São Paulo (1 cidade) & Mata Atlântica & Fragmento florestal & Dáttilo et al., 2011 \\
\hline & $\begin{array}{c}\text { Ponerinae } \\
\text { ('Pachycondyla'sp.) (1 } \\
\text { espécie) }\end{array}$ & São Paulo (1 cidade) & Mata Atlântica & Hospital & Zarzuela et al., 2002 \\
\hline & $\begin{array}{c}\text { Ponerinae } \\
\text { ('Pachycondyla'sp.) (1 } \\
\text { espécie) }\end{array}$ & Bahia (1 cidade) & Mata Atlântica & Fragmento florestal & $\begin{array}{l}\text { Guimarães et al., } \\
2013\end{array}$ \\
\hline & $\begin{array}{c}\text { Ponerinae } \\
\text { ('Pachycondyla'spp.) } \\
\text { (2 espécies) }\end{array}$ & São Paulo (1 cidade) & Mata Atlântica & Casa & Kamura et al., 2007 \\
\hline & $\begin{array}{c}\text { Ponerinae } \\
\text { ('Pachycondyla'spp.) } \\
\text { (2 espécies) }\end{array}$ & São Paulo (1 cidade) & Mata Atlântica & Casa & Piva; Campos, 2012 \\
\hline
\end{tabular}




\begin{tabular}{|c|c|c|c|c|c|}
\hline SUBFAMÍLIA & ESPÉCIE & ESTADO & BIOMA & HABITAT & AUTOR \\
\hline & $\begin{array}{c}\text { Ponerinae } \\
\text { ('Pachycondyla'spp.) } \\
\text { (3 espécies) }\end{array}$ & Bahia (1 cidade) & Mata Atlântica & $\begin{array}{l}\text { Ambiente urbano } \\
\text { não especificado }\end{array}$ & Delabie et al., 1999 \\
\hline & $\begin{array}{c}\text { Ponerinae } \\
\text { ('Pachycondyla'spp.) } \\
\text { (3 espécies) }\end{array}$ & Paraná (1 cidade) & Mata Atlântica & Casa e indústria & $\begin{array}{l}\text { Oliveira; Campos- } \\
\text { Farinha, } 2005\end{array}$ \\
\hline & $\begin{array}{c}\text { Rasopone arhuaca } \\
\text { (Forel, 1901) }\end{array}$ & Bahia (1 cidade) & Mata Atlântica & Fragmento florestal & Melo et al., 2014 \\
\hline & $\begin{array}{c}\text { Rasopone ferruginea } \\
\text { (Smith, 1858) }\end{array}$ & Bahia (1 cidade) & Mata Atlântica & Fragmento florestal & Melo et al., 2014 \\
\hline & Thaumatomyrmex sp. & Bahia (3 cidades) & Mata Atlântica & Fragmento florestal & Melo et al., 2014 \\
\hline Proceratiinae & $\begin{array}{c}\text { Discothyrea } \\
\text { sexarticulata } \\
\text { (Borgmeier, 1954) }\end{array}$ & São Paulo (1 cidade) & Mata Atlântica & Fragmento florestal & Morini et al., 2007 \\
\hline
\end{tabular}

Observação: 'Pachycondyla'spp. correspondem a diversos Ponerinae, antes da revisão de Schmidt; Shattuck (2014).

No Brasil, se destacam como formigas invasoras de ambientes urbanos: Monomorium floricola Jerdon, 1851, Monomorium pharaonis Linnaeus, 1758, Paratrechina longicornis Latreille, 1802, Pheidole megacephala Fabricius, 1793, Solenopsis geminata (Fabricius, 1804), Solenopsis invicta Buren, 1972, Solenopsis saevissima Smith, 1855, Tapinoma melanocephalum Fabricius, 1793, Tetramorium bicarinatum Nylander, 1846, Tetramorium simillimum Smith, 1851 e Wasmannia auropunctata Roger, 1863 (DELABIE, et al., 1995; MARQUES et al., 2002; OLIVEIRA; CAMPOS-FARINHA, 2005; KAMURA et al., 2007; PACHECO; VASCONCELOS, 2007; PESQUERO et al., 2008; MUNHAE et al., 2009; DÁTTILO et al., 2011; PIVA; CARVALHO-CAMPOS, 2012; MELO et al., 2014). Destas, somente as Solenopsis spp. e $W$. auropunctata são nativas, não havendo registro de espécies exóticas de formigas poneromorfas (exemplo: Hypoponera punctatissima (Roger, 1859) e Hypoponera opacior Forel, 1893) para as cidades brasileiras. Apesar de H. punctatissima e H. opacior serem introduzidas no Brasil, estarem presentes em ambientes antropizados, estas espécies não foram encontradas em ambiente urbano (DELABIE; BLARD, 2002; LUTINSKI et al., 2008) não podendo ser consideradas formigas sinantrópicas. Apesar de termos encontrado um grande número de espécies nativas de poneromorfas listadas para cidades do Brasil, não encontramos referências que afirmam que alguma das formigas listadas nesse levantamento possua hábitos sinantrópicos. Contudo, propomos que as espécies E. edentatum, G. striatula, H. distinguenda, $P$. harpax e $P$. striata sejam consideradas sinantrópicas, devido à grande variedade de habitats urbanos que são capazes de ocupar.

\section{Referências}

AGUILERA, F.; VALENZUELA, L. M.; BOTEQUILHALEITÃO, A. Landscape metrics in the analysis of urban land use patterns: A case study in a Spanish metropolitan area. Landscape and Urban Planning, 99: 226-238, 2011.

ALBERTI, M. The effects of urban patterns on ecosystem function. International Regional Science Review, 2: 168-192, 2005.

ANTONOV, I. A. Ant assemblages of two cities with different ecological conditions in Southern Cisbaikalia. Russian Journal of Ecology, 39: 454-456, 2008.

AQUINO, R. S. S.; SILVEIRA, S. S.; PESSOA, W. F. B.; RODRIGUES, A.; ANDRIOLI, J. L.; DELABIE, J. H. C.; FONTANA, R. Filamentous fungi vectored by ants (Hymenoptera: Formicidae) in a public hospital in north-eastern Brazil. Journal of Hospital Infection, 83: 200-204, 2012.

BUCZKOWSKI, G. Suburban sprawl: environmental features affect colony social and spatial structure in the black carpenter ant, Camponotus pennsylvanicus. Ecological Entomology, 36: 62-71, 2011.

BUCZKOWSKI, G.; RICHMOND, D. S. The Effect of Urbanization on Ant Abundance and Diversity: A Temporal Examination of Factors Affecting Biodiversity. PLoS OneE, 7: 1-9, 2012

BYRNE, L.B. Habitat structure: A fundamental concept and framework for urban soil ecology. Urban Ecosystems, 10: 255-274, 2007.

CANTONE, S.; CAMPOS, A. E. C. Diversity of Ponerinae ants (Hymenoptera: Formicidae) using urban areas for reproduction: their potential threat to human health. Proceedings of the Eighth International Conference on Urban Pests, 253-258, 2014. 
CLARKE, K. M.; FISHER, B. L.; LEBUHN, G. The influence of urban park characteristics on ant (Hymenoptera, Formicidae) communities. Urban Ecosystems, 11: 317-334, 2008.

DÁTTILO, W.; SIBINEL, N.; FALCÃO, J. C. F.; NUNES, R. V. Ant fauna in a urban remnant of Atlantic Forest in the municipality of Marília, state of São Paulo, Brazil. Bioscience Journal, 27: 494-504, 2011.

DEARBORN, D. C.; KARK, S. Motivations for Conserving Urban Biodiversity. Conservation Biology, 24: 432-440, 2010.

DELABIE, J. H. C; NASCMENTO, I. C.; PACHECO, P.; CASIMIRO, A. B. Community structure of houseinfesting ants (Hymenoptera: Formicidae) in Southern Bahia, Brazil. Florida Entomologist, 78: 264-270, 1995.

DELABIE, J. H. C; NASCIMENTO, I. C.; MARIANO, C. S. F. Importance de l'agricul ture cacaoyère pour le maintien de la biodiversité: étude comparée de la myrmecofaune de différents milieux du sud-est de Bahia, Brésil (Hymenotptera; Formicidae). 12 ${ }^{\text {th }}$ International Cocoa Research Conference, 23-30, 1999.

DELABIE, J. H. C; BLARD F. The tramp ant Hypoponera punctatissima (Roger) (Hymenoptera: Formicidae: Ponerinae): new records from the Southern Hemisphere. Neotropical Entomology, 31: 149-151, 2002.

DELABIE, J. H. C.; CÉRÉGHINO, R.; GROC, S.; DEJEAN, A.; GIBERNAU, M.; CORBARA, B.; DEJEAN, A. Ants as biological indicators of Wayana Amerindian land use in French Guiana. C. R. Biologies, 332: 673-684, 2009.

DIEHL-FLEIG, E. Formigas invasoras: o caso da formiga argentina Linepithema Humile (Mayr 1868). Acta Biologica Leopondensia, 28:5-9, 2006.

FAETH, S. H.; BANG, C.; SAARI1, S. Urban biodiversity: patterns and mechanisms. Annals of New York Academy of Sciences, 1223: 69-81, 2011.

FARNEDA, F. Z.; LUTINSKI, J. A.; GARCIA, F. R. M. Comunidade de formigas (Hymenoptera: Formicidae) na área urbana do município de Pinhalzinho, Santa Catarina, Brasil. Revista de Ciências Ambientais, 1: 53-66, 2007.

FRIEDRICH, R.; PHILPOTT, S. M. Nest-site limitation and nesting resources of ants (Hymenoptera: Formicidae) in urban green spaces. Environmental Entomology, 38: 600-607, 2009.
GARCIA, F. R. M.; AHLERT, C. C.; FREITAS, B. R.; TRAUTMANN, M. M.; TANCREDO, S. P.; LUTINSKI, J. A. Ants (Hymenoptera: Formicidae) in five hospitals of Porto Alegre, Rio Grande do Sul State, Brazil. Acta Scientiarum, 33: 203-209, 2011.

GRIMM, N. B.; FAETH, S. H.; GOLUBIEWSKI, N. E.; REDMAN, C. L.; WU, J.; BAI, X.; BRIGGS, J. M. Global Change and the Ecology of Cities. Science, 319: 756-760, 2008.

GUÉRNARD, B.; CASAS, A. C.; DUNN, R. R. High diversity in an urban habitat: are some animal assemblages resilient to long-term anthropogenic change?. Urban Ecosystems, 1-15, 2014.

GUIMARÃES, M. V. A.; BENATI, K. R.; PERES, M. C. L.; DELABIE, J. H. C. Assembléia de formigas de serrapilheira em fragmentos florestais no município de Salvador, Bahia, Brasil. Biociências, 19: 1-9, 2013.

GUZMÁN-MENDOZA, R.; CASTAÑO-MENESES, G.; HERRERA-FUENTES, M. C. Variación espacial y temporal de la diversidad de hormigas en el Jardín Botánico del valle de Zapotitlán de las Salinas, Puebla. Revista Mexicana de Biodiversidad, 81: 427-435, 2010.

HOLWAY, D. A.; SUAREZ, A. V. Homogenization of ant communities in Mediterranean California: The effects of urbanization and invasion. Biological Conservation, 127: 319-326, 2006.

IBGE - INSTITUTO BRASILEIRO DE GEOGRAFIA E ESTATÍSTICA. 2012. Países. Acessado em 11/02/14. Disponível em http://www. ibge.gov.br/paisesat/

IOP, S.; CALDART, V. M.; LUTINSKI, J. A.; GRACIA, F. R. M. Formigas urbanas da cidade de Xanxerê, Santa Catarina, Brasil. Biotemas, 22: 55-64, 2009.

IVANOV, K.; KEIPER, J. Ant (Hymenoptera: Formicidae) diversity and community composition along sharp urban forest edges. Biodiversity Conservation, 19:3917-3933, 2010.

KAMURA, C. M.; MORINI, M. S. C.; FIGUEIREDO, C. J.; BUENO, O. C.; CAMPOSFARINHA, A. E. C. Ant communities (Hymenoptera: Formicidae) in an urban ecosystem near the Atlantic Rainforest. Brazilian Journal of Biology, 67: 635-641.

KOWARIK, I. Novel urban ecosystems, biodiversity, and conservation. Environmental Pollution, 159: 1974-1983, 2011. 
KUMAR, D.; MISHRA, A. Ant community variation in urban and agricultural ecosystems in Vadodara District (Gujarat State), western India. Asian Myrmecology, 2: 85-93, 2008.

LESSARD, J. P.; BUDDLE, C. M. The effects of urbanization on ant assemblages (Hymenoptera: Formicidae) associated with the Molson Nature Reserve, Quebec. Canadian Entomologist, 137: 215225, 2005.

LOPES, A. S. Comunidades de Formigas (Hymenoptera: Formicidae), em Área Urbana e em Área Rural da cidade de Sorocaba / SP. Revista Eletrônica de Biologia, 2: 32-46, 2009.

LUNDHOLM, J. T.; RICHARDSON, P. J. Habitat analogues for reconciliation ecology in urban and industrial environments. Journal of Applied Ecology, 47: 966-975, 2010.

LUTINSKI, J. A., GARCIA, F. R. M.; LUTINSKI, C. J.; IOP, S. Diversidade de formigas na Floresta Nacional de Chapecó, Santa Catarina, Brasil. Ciência Rural, 38 : 1810-1816, 2008.

LUTINSKI, J. A.; LOPES, B. C.; MORAIS, A. B. B. Urban ant diversity (Hymenoptera: Formicidae) in tem cities of southern Brazil. Biota Neotropical, 13: 332$342,2013$.

MARQUES, A. P. C.; ALE-ROCHA, R.; RAFAEL, J. A. Levantamento de formigas (Hymenoptera: Formicidae) em residências de Manaus, estado do Amazonas, Brasil. Acta Amazonica, 32: 133-140, 2002.

MCINTYRE, N. E.; KNOWLES-YÁNEZ, K.; HOPE, D. Urban ecology as an interdisciplinary field: differences in the use of "urban" between the social and natural sciences. Urban Ecosystems, 4: 5-24, 2000.

MCINTYRE, N. E.; RANGO, J.; FAGAN, W. F; FAETH, S. H. 2001. Ground arthropod community structure in a heterogeneous urban environment. Landscape and Urban Planning, 52: 257-274, 2000.

MCKINNEY, M. L. Urbanization, Biodiversity, and Conservation. BioScience, 52: 883-890, 2002.

MCKINNEY, M. L. Urbanization as a major cause of biotic homogenization. Biological Conservation, 127: 247-260, 2006.

MELO, T. S.; PERES, M. C. L.; CHAVARI, J. L.; BRESCOVIT, A. D.; DELABIE, J. H. C. Ants (Formicidae) and Spiders (Araneae) listed from the Metropolitan Region of Salvador, Brazil. Check List, 10: 355-365, 2014.
MENKE, S. B.; BOOTH, W.; DUNN, R. R.; SCHAL, C.; VARGO, E. L.; SILVERMAN, J. Is It Easy to Be Urban? Convergent Success in Urban Habitats among Lineages of a Widespread Native Ant. Plos One, 5: $1-9,2010$.

MENKE, S. B.; GUÉNARD, B.; SEXTON, J. O.; WEISER, M. D.; DUNN, R. R.; SILVERMAN, J. Urban areas may serve as habitat and corridors for dryadapted, heat tolerant species; an example from ants. Urban Ecosystems, 14:135-163, 2011.

MORINI, M. S. C.; MUNHAE, C. B.; LEUNG, R.; CANDIANI, D. F. ; VOLTOLINI, J. C. Comunidades de formigas (Hymenoptera, Formicidae) em fragmentos de Mata Atlântica situados em áreas urbanizadas. Iheringia, 97: 246-252, 2007.

MUNHAE, C. B.; BUENO, Z. A. F. N.; MORINI, M. S. C.; SILVA, R. R. Composition of the Ant Fauna (Hymenoptera: Formicidae) in Public Squares in Southern Brazil. Sociobiology, 53: 455-472, 2009.

OLIVEIRA, M. F.; CAMPOS-FARINHA, A. E. C. Formigas urbanas do município de Maringá, $\mathrm{PR}$, e suas implicações. Arquivos do Instituto Biológico, 72: 33 $39,2005$.

ONU - ORGANIZAÇÃO DAS NAÇÕES UNIDAS. 2011. World Urbanization Prospects. Acessado em 11/02/14. Disponível em http://www.un.org/en/ development/desa/population/

PACHECO, R.; VASCONCELOS, H. L. Invertebrate conservation in urban areas: Ants in the Brazilian Cerrado. Landscape and Urban Planning, 81: 193 199, 2007.

PASQUEIRO, M. A.; FILHO, J. E.; CARNEIRO L. C.; FEITOSA S. B.; OLIVEIRA, M. A. C.; QUINTANA, R. C. Formigas em Ambiente Hospitalar e seu Potencial como Transmissoras de Bactérias. Neotropical Entomology, 37: 472-477, 2008.

PECAREVIC, M.; DANOFF-BURG, J.; DUNN, R. R. Biodiversity on Broadway - Enigmatic Diversity of the Societies of Ants (Formicidae) on the Streets of New York City. PLoS One, 5: 1-8, 2010.

PICKETT, S. T. A.; CADENASSO, M. L. Advancing urban ecological studies: frameworks, concepts, and results from the Baltimore ecosystem study. Austral Ecology, 31: 114-125, 2006.

PIVA, A.; CAMPOS, A. E. C. Ant community structure (Hymenoptera: Formicidae) in two neighborhoods with different urban profiles in the city of São Paulo, Brazil. Psyche, 1: 1-8, 2012. 
SANFORD, M. P.; MANLEY, P. N.; MURPHY, D. D. Effects of Urban Development on Ant Communities: Implications for Ecosystem Services and Management. Conservation Biology, 23: 131-141, 2009.

SATTLER, T.; DUELLI, P.; OBRIST, M. K.; ARLETTAZ, R.; MORETTI, M. Response of arthropod species richness and functional groups to urban habitat structure and management. Landscape Ecology, 25: 941-954, 2010.

SCHMIDT, C. A.; SHATTUCK, S. O. The higher classification of the ant subfamily Ponerinae (Hymenoptera: Formicidae), with a review of ponerine ecology and behavior. Zootaxa, v. 3817, n. 1, p. 1-242, 2014. Disponível em: <http://www.ncbi.nlm.nih.gov/ pubmed/24943802>.

SHOCHAT, E.; WARREN, P. S.; FAETH, S. H.; MCINTYRE, N. E.; HOPE, D. From patterns to emerging processes in mechanistic urban ecology. Trends in Ecology and Evolution, 21: 186-191, 2006.

SOARES, N. S.; ALMEIDA, L. O.; GONÇALVES, C. A.; MARCOLINO, M. T.; BONETTI, A. M. Levantamento da Diversidade de Formigas (Hymenoptera: Formicidae) na Região Urbana de Uberlândia, MG. Neotropical Entomology, 35: 324-328

SOUZA, D. R.; MUNHAE, C. B.; KAMURA, C. M; PORTERO, N. S.; MORINI M. S. C. Formigas em áreas urbanizadas da Serra do Itapeti. In: Morini, M.S.C.; Miranda, V. F. O. Serra do Itapeti, Aspectos Históricos, Sociais e Naturalísticos, p. 301-308, 2012.
TOENNISSON, T. A.; SANDERS, N. J.; KLINGEMAN, W. E.; VAIL, K. M. Influences on the Structure of Suburban Ant (Hymenoptera: Formicidae) Communities and the Abundance of Tapinoma sessile. Environmental Entomology, 40: 1397-1404, 2011.

UNO, S.; COTTON, J.; PHILPOTT, S. M. Diversity, abundance, and species composition of ants in urban green spaces. Urban Ecosystems, 13: 425-441, 2010.

VEPSALAINEN, K.; IKONEN, H.; KOIVULA, M. $\mathrm{J}$. The structure of ant assemblages in an urban area of Helsinki, southern Finland. Annales Zoologici Fennici, 45: 109-127, 2008.

VITOUSEK, P. M.; MOONEY, H. A.; LUBCHENCO, J.; MELILLO, J. M. Human Domination of Earth's Ecosystems. Science, 277: 494-499, 1997.

WETTERER, J. K.; DUTRA, D. S. Ants on Sapling Cecropia in Forest and Urban Areas of Trinidad (Hymenoptera: Formicidae). Sociobiology, 58: 17-22, 2011.

YAMAGUCHI, T. Influence of urbanization on ant distribution in parks of Tokyo and Chiba City, Japan. Ecological Research, 19: 209-216, 2004.

ZARZUELA, M. F. M.; RIBEIRO, M. C. C.; CAMPOSFARINHA, A. E. C. Distribuição de formigas urbanas em um hospital da região sudeste do Brasil. Arquivos do Instituto Biológico, 69: 85-87, 2002. 